\title{
Rapid molecular detection of tuberculosis and rifampicin drug resistance: retrospective analysis of a national UK molecular service over the last decade
}

\author{
N Seoudi, ${ }^{1,2}$ S L Mitchell, ${ }^{1,3}$ T J Brown, ${ }^{1,3}$ F Dashti, ${ }^{2}$ A K Amin, ${ }^{2}$ F A Drobniewski ${ }^{1,2,3}$
}

- Additional appendices are published online only. To view these files please visit the journal online (http://thorax.bmj. com/content/67/4.toc)

${ }^{1}$ Blizard Institute, Barts and the London School of Medicine and Dentistry, Queen Mary University, London, UK 2Department of Medical Microbiology, Barts and the London NHS Trust, London, UK ${ }^{3}$ National Mycobacterium Reference Laboratory, Health Protection Agency, London, UK

\section{Correspondence to}

Professor F A Drobniewski, HPA National Mycobacterium Reference Laboratory, Clinical TB and HIV Group, Blizard Institute, 2 Newark Street, London E1 2AT, UK

f.drobniewski@qmul.ac.uk

Received 12 June 2011 Accepted 25 November 2011 Published Online First 2 January 2012

\begin{abstract}
Background Fast and reliable detection of Mycobacterium tuberculosis complex (MTBC) and drug resistance is crucial in establishing effective treatment and enforcing timely public health measures.
\end{abstract}

Methods The authors analysed the performance of a national UK molecular diagnostic service over a decade, based on the use of a line probe assay (Innolipa, LiPA) compared with conventional liquid and solid cultures with rapid molecular identification and culture-based drug resistance testing.

Findings Data were available for 7836 consecutive patient samples using LiPA and the reference microbiological technique (conventional liquid and solid cultures with rapid molecular identification and culturebased drug resistance testing). For all sputum specimens ( $n=3382$ ) the sensitivity, specificity, positive predictive value, negative predictive value and accuracy for MTBC detection were $93.4 \%, 85.6 \%, 92.7 \%, 86.9 \%$ and $90.7 \%$; the equivalent values for smear-positive sputum specimens ( $\mathrm{n}=2606)$ were $94.7 \%, 80.9 \%, 93.9 \%, 83.3 \%$ and $91.3 \%$. The sensitivity, specificity, positive predictive value, negative predictive value and accuracy for detection of rifampicin resistance in all sputum samples $(n=1667)$ were $92.1 \%, 99.3 \%, 89.4 \%, 99.5 \%$ and $98.9 \%$, respectively; the equivalent values for smearpositive sputum specimens $(n=1477)$ were $93.3 \%$, $99.3 \%, 87.5 \%, 99.6 \%$ and 99\%. Between January 2006 and December 2008, LiPA saved 25.3 and 32.2 days for TB diagnosis and rifampicin resistance of smear-positive samples, respectively.

Interpretation A molecular diagnostic service, using a non-automated line probe assay approach, provides a rapid and reliable national service for diagnosing MTBC and rifampicin resistance.

\section{INTRODUCTION}

The rapid identification of multidrug resistant tuberculosis (MDRTB) (ie, tuberculosis (TB) isolates resistant to at least isoniazid and rifampicin) reduces the time for the instigation of appropriate treatment, helps to reduce the spread of drug-resistant TB and may improve survival. ${ }^{1-4}$

Conventional drug susceptibility testing (DST) can take 1-2 weeks once a positive culture has been obtained. In recent years, the use of assays for the genetic detection of mutations that confer resistance have been developed and evaluated. Among these, line probe assays (LPAs) are viewed as a rapid, very specific and sensitive tool for the detection of multidrug-resistant TB. ${ }^{5}$ Moreover, the
WHO have recently endorsed a policy of the use of LPAs for the rapid screening of patients at risk of $\mathrm{MDRTB}^{6}$ and in 2010 recommended the use of the GeneXpert, XpertMTB/RIF following a successful multicentre analysis. ${ }^{7}$

In 1998, national and population-based services were proposed for the diagnosis of $\mathrm{TB}$ and rifampicin resistance directly from smear-positive patient specimens, ${ }^{8}$ and these were adopted in the UK and in other countries using LPAs. The UK Health Protection Agency National Mycobacterium Reference Laboratory (HPA NMRL) implemented this national molecular TB diagnostic service (branded as 'Fastrack') in 1999-2000 with promising initial results. ${ }^{5}$ Rapid identification of rifampicin resistance and MDRTB with automated liquid culture is performed at the same time; specimens identified as MDRTB have any resulting cultures analysed for all first-line and second-line drugs simultaneously.

Using a different LPA, excellent sensitivity, specificity, positive predictive value (PPV) and negative predictive (NPV) were obtained when used directly on 536 smear-positive sputum specimens in South Africa, ${ }^{9}$ demonstrating that these techniques worked well in low and middle income countries.

The study evaluates the accuracy of results obtained for TB diagnosis and rifampicin detection compared with rapid culture-based methods in the context of a routine, non-trial national molecular diagnostic service. Sensitivity, specificity, PPV and NPV, analysis of discrepant results and turnaround times were obtained and compared with our previously published data to underline a decade of experience of these assays.

This study complements other published studies on the automated GeneXpert system and its XpertMTB/RIF assay $^{7}$ by providing extensive evidence for an alternative system of diagnosis supporting WHO policy statements on the use of both LPAs and XpertMTB/RIF, and demonstrating the value of a national operational service.

\section{MATERIALS AND METHODS \\ Clinical specimens}

A total of 8501 consecutive primary specimens were referred to the UK HPA NMRL at the request of National Health Service (NHS) physicians over a 7-year period (January 2003-December 2009) for molecular 'Fastrack' analysis. A single specimen from each patient was analysed in any one year The reference assay was conventional culture-based diagnosis and rifampicin resistance (see below) 
Out of the 7836 samples with both molecular and comparative culture-based results, 4420 were respiratory samples, including 3382 sputum samples, 733 bronchoalveolar lavage (BAL) samples, 269 pleural fluid aspirates and 36 other respiratory samples such as lung biopsy; 3406 samples were non-respiratory, including 1638 cerebrospinal fluid (CSF) samples.

\section{Microscopy, culture and susceptibility testing}

All samples were decontaminated and processed according to the NMRL standard operating procedure, which has previously been described. $^{5} \mathrm{NaOH} / \mathrm{N}$ acetyl-L-cysteine ( $\left.\mathrm{NaOH} / \mathrm{NALC}\right)(6 \mathrm{ml})$ was added to each sample in a $50 \mathrm{ml}$ Falcon tube. The sample was allowed to be incubated with the $\mathrm{NaOH} / \mathrm{NALC}$ for $30 \mathrm{~min}$ with periodic gentle mixing. Subsequently, each sample was diluted (1:40 dilution) with phosphate buffer ( $\mathrm{pH}$ 6.8) followed by centrifugation at $3000 \times \mathrm{g}$ for $30 \mathrm{~min}$. The supernatant was discarded and $2 \mathrm{ml}$ of sterile phosphate buffer was added to resuspend the pellet. Half of the suspension was used for the molecular assay, $0.25 \mathrm{ml}$ of each decontaminated sample was cultured on Lowenstein-Jensen (LJ) slopes and $0.5 \mathrm{ml}$ was inoculated into mycobacterial growth indicator tubes (MGIT) (Becton, Dickinson and Company, New Jersey, USA). Suitable samples for microscopy examination were investigated by fluorescent auramine phenol staining according to the NMRL standard operating procedure.

First-line DST was carried out on all culture-positive MTBC isolates using the resistance ratio method on LJ slopes ${ }^{10}$ and including rifampicin, isoniazid, ethambutol and pyrazinamide. Second-line (reserve) DST was performed using the MGIT system. The isolated microorganism was identified by using GenoType-Series molecular assay (Hain Lifescience $\mathrm{GmbH}$, Nehren, Germany).

\section{LiPA}

DNA was extracted from $1 \mathrm{ml}$ of each decontaminated sample and a commercial and validated LPA assay (Innolipa, LiPA; Innogenetics, Ghent, Belgium) was performed for all samples included in the analyses (7836 specimens) according to the manufacturer's instructions and as previously described. ${ }^{5}$

\section{Standardisation and blinding}

Staff performing molecular analyses were blind to the culturebased reference methods. Bacteriological staff usually had no prior experience of molecular diagnostic assays but were appropriately trained to perform the LPA over a 1-week period using written standard operating procedures. $\mathrm{A} \mathrm{PhD}$ grade molecular scientist oversaw the service. Culture-based internal and external quality assurance and proficiency systems were in place throughout and were introduced in 2003 for molecular assays.

\section{Statistical analysis}

Data were entered into a Microsoft Excel spreadsheet (Microsoft Corporation, Washington, USA) and analysed by Prism (GraphPad, USA). The accuracy of the LiPA assay for detecting MTBC and rifampicin resistance was compared with the accepted standards of culture (ie, one MGIT plus one LJ slope), and phenotypic DST. ${ }^{11} 12$ Sensitivity, specificity, PPV, NPV and diagnostic accuracy were calculated as follows: sensitivity $(\%)=$ (true positive/true positive + false negative) $\times 100$; specificity $(\%)=($ true negative/false positive + true negative $) \times 100 ;$ PPV $(\%)=($ true positive/true positive + false positive $) \times 100 ; \mathrm{NPV}$ $(\%)=($ true negative/false negative + true negative $) \times 100 ;$ and diagnostic accuracy $(\%)=$ (true positive + true negative/total number) $\times 100$. An increase in sensitivity indicated a decrease in false-negative cases, while an increase in specificity indicated a decrease in false-positive cases. PPV is defined as the probability that a person has the disease and their test was positive, while NPV is defined as the probability that a person does not have the disease and their test was negative. CIs were calculated using the method of Vollset. ${ }^{13}$ The sensitivity, specificity, PPV and NPV were compared (using the $\chi^{2}$ test) with our previously published data. ${ }^{5}$ Statistical significance was determined using a $p$ value $\leq 0.05$.

Detailed turnaround times were calculated for a recent 3-year period (2006-8). The time to diagnosis was calculated from the date of receiving the sample in the NMRL to the date of issuing the results to the clinician. The time to diagnosis was based on direct LiPA testing of patient specimens compared with the time taken for culture growth and identification of the isolated mycobacterium. The NMRL use GenoType-Series molecular assays for rapid identification of mycobacterial cultures (performed daily, Monday-Friday).

\section{RESULTS}

Of the 8501 consecutive samples received by the NMRL for LiPA assay during the period between January 2003 and December 2009, comparison with bacteriological culture was possible for 7836 samples (culture could not be performed or was contaminated in 290 (3.4\%) samples; PCR was not performed for 140 $(1.6 \%)$ samples (see below); PCR was inhibited or not interpretable in 285 (3.4\%) samples (including 50 specimens which also had contaminated cultures); hence these were excluded from the analysis) (figure 1).
Figure 1 Flow chart of samples received in the National Mycobacterium Reference Laboratory (NMRL) in the period between January 2003 and December 2009. AFB, acid fast bacilli; +ve, positive; -ve, negative.

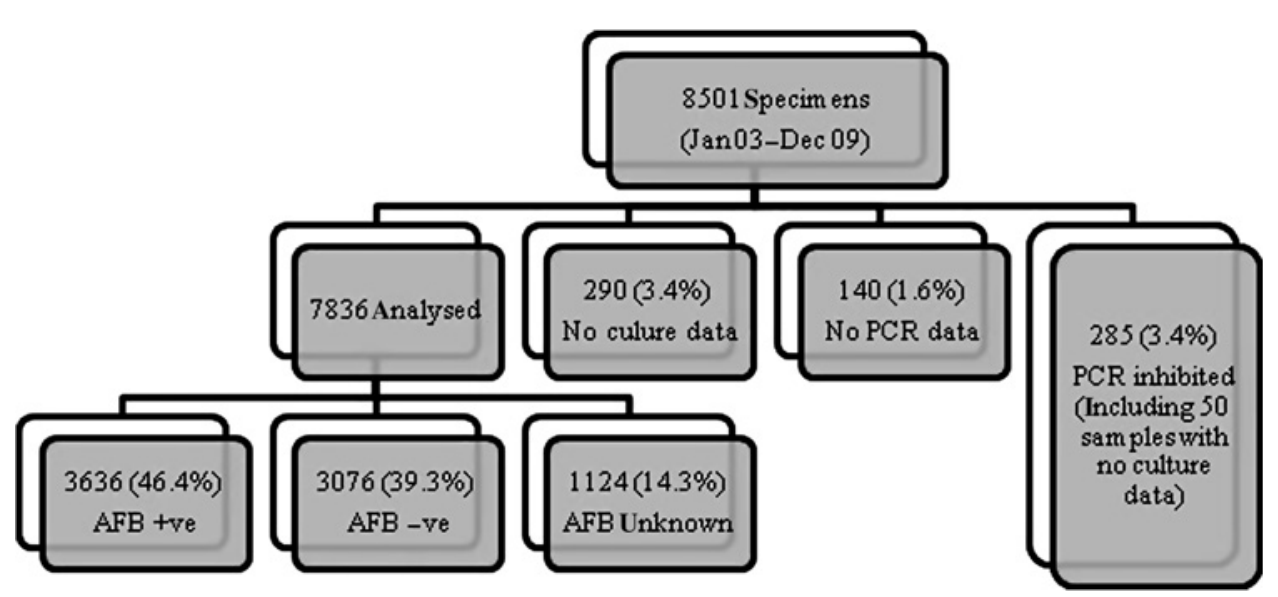


Specimens were analysed by sample type and subdivided by microscopy result if known. Specimens were also analysed as 'respiratory' (sputum, BAL, pleural fluid and other respiratory samples such as lung biopsy) and 'non-respiratory' (CSF, lymph node biopsy, aspirate, pus, blood, pericardial fluid and others including urine and other biopsies) and subdivided by microscopy result if known (tables 1,2 and supplementary online tables A1-A2).

The acid-fast bacilli smear microscopy was positive for 3636 of 7836 primary specimens (46.4\%), negative for 3076 (39.3\%) and unknown for 1124 (14.3\%). These 1124 specimens that did not have microscopy performed routinely or did not have a microscopy result were included in the main analysis but excluded from the specific sub-analysis described in tables 1-2 and online supplementary tables A1-A2. A total of 3299 MTBC and 320 non-tuberculosis mycobacteria (NTM) were cultured in the 7-year study period.

\section{LiPA}

LiPA was not performed in 140 of 8501 (1.6\%) specimens as a culture had been sent previously and the MTBC and rifampicin results were already known. For the 8361 processed samples, the results of LiPA analysis for MTBC were negative for 4745 (56.8\%), positive for 3331 (39.8\%), and 285 (3.4\%) were inhibited or not interpretable. Of the 3331 PCR-positive cases, $146(4.4 \%)$ were reported as rifampicin resistant, $3173(95.2 \%)$ were reported as rifampicin susceptible and 12 (0.4\%) gave equivocal results.

The overall sensitivity, specificity, PPV, NPV and diagnostic accuracy of LiPA assay for MTBC detection were analysed using different sample types in relation to the standard culture-based technique as shown in table 1 and online supplementary table A1. Overall sensitivity, specificity, PPV, NPV and diagnostic accuracy of rifampicin resistance in comparison to the standard resistance ratio drug susceptibility method (phenotypic sensitivity) are shown in table 2 and online supplementary table A2.

A single molecular test performed against liquid and solid culture for all specimens had an overall sensitivity of $87.3 \%$, specificity of $91.1 \%$, PPV of $87.7 \%$, NPV of $90.8 \%$ and overall accuracy of $89.5 \%$ for MTBC detection (table 1 ). For all sputum specimens $(n=3382)$ the sensitivity, specificity, PPV, NPV and accuracy, in comparison with specimens that became culture positive, were $93.4 \%, 85.6 \%, 92.7 \%, 86.9 \%$ and $90.7 \%$, respectively. The equivalent figures for smear-positive specimens $(\mathrm{n}=2606)$ were $94.7 \%, 80.9 \%, 93.9 \%, 83.3 \%$ and $91.3 \%$ and for smear-negative specimens were $77.8 \%, 92.7 \%, 77.2 \%, 92.9 \%$ and $89.1 \%$, respectively. The results for non-sputum samples were good (with the exception of CSF), although with lower sensitivity, for example, BAL smear-positive sensitivity was $86.8 \%$ (online supplementary table A1).

The DST data were available for 2306 specimens for both techniques (LiPA and the standard resistance ratio susceptibility method). A total of 136 (5.9\%) specimens were reported as rifampicin resistant by LiPA and 2170 (94.1\%) were sensitive. In contrast, $132(5.7 \%)$ specimens were reported as rifampicin resistant using the standard resistance ratio susceptibility method and 2174 (94.3\%) were sensitive.

The overall sensitivity, specificity, PPV, NPV and accuracy for the detection of rifampicin resistance for all specimens which became culture positive and for which comparative phenotypic DST data were available $(\mathrm{n}=2306)$, was as follows: $92.4 \%$, $99.4 \%, 89.7 \%, 99.5 \%$ and $99.0 \%$, respectively. Out of the 2306 samples analysed; the acid-fast bacilli smear microscopy was

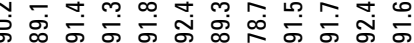

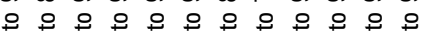
œ

๘



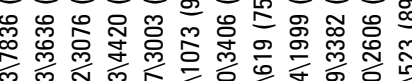

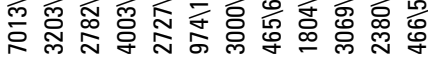

c.

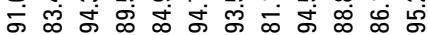

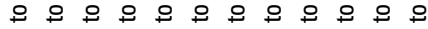

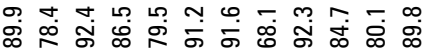


$\infty$ ก

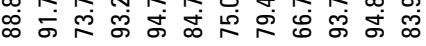

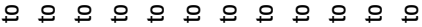
䎹

o क व

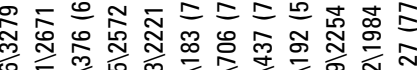

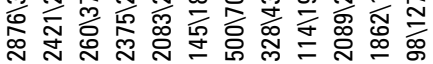

の m $m$ m

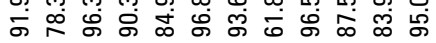

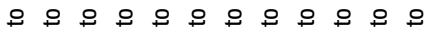
궁

* の 0 \%

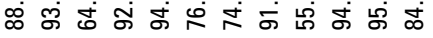
옹ㅇㅇㅇㅇㅇㅇㅇㅇㅇㅇㅇㅇ ঢ் 
๗ 옹ㅇㅇㅇㅇㅇㅇㅇㅇㅇㅇㅇ (1)



จ

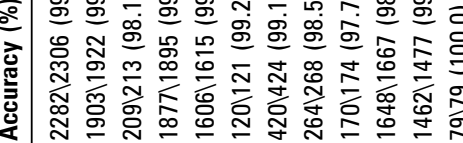


엉ㅇㅇㅇㅇㅇㅇㅇㅇㅇㅇㅇㅇㅇ

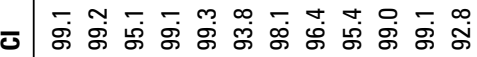

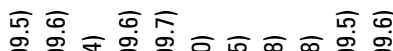

o

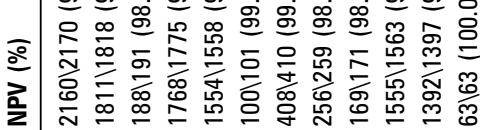

일

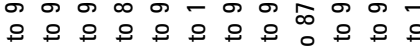

ভ

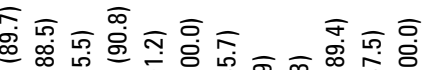
๑

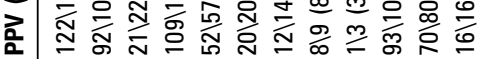

๑ 6 웅 용 용 용 용 용 용 용 으



उ

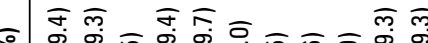

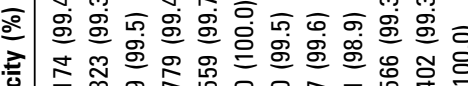

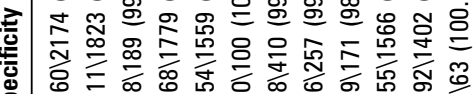

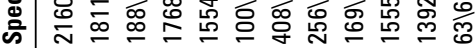

- 0 -


응ㅇㅇㅇㅇㅇㅇㅇㅇㅇㅇㅇㅇㅇ - L

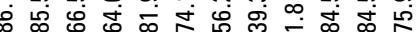

๑ே

于 0 a 崩



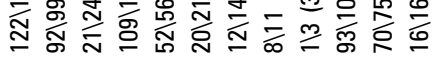
政 positive for 1922 primary specimens (1922/2306) (83.3\%) negative for 213 (213/2306) (9.2\%) and unknown for 171 (171/ 2306) (7.4\%); table 2 and online supplementary table A2).

In the period between January 2006 and December 2008, implementation of molecular techniques enabled an earlier diagnosis for all samples and saved a mean of 35.9 days compared with culture. Focusing on all smear-positive samples only, the assay consistently helped to reach an earlier diagnosis of MTBC and rifampicin resistance (25.3 \pm 19.4 and $32.2 \pm 16.9$ days saved, respectively) (table 3 ).

\section{Discrepant results}

In the period 2003-2009, there were 420 false-negative results for MTBC; these samples were found to be negative using LiPA but culture positive for MTBC. Of these 420 false-negative results, $220(52.3 \%)$ specimens were respiratory and 200 (47.6\%) were non-respiratory. Of 403 false-positive MTBC results (specimens positive using PCR but failing to grow on microbiological culture), 197 (48.9\%) were respiratory samples and 206 (51.1\%) were nonrespiratory samples. Interestingly, only eight cases out of the total of 320 NTM (2.5\%) cultured using standard techniques were positive using LiPA and accounted for $<2 \%$ of the false-positive cases. We believe that these eight cases were mixed MTBC and NTM or contaminated cultures but no MTBC was cultured probably due to overgrowth by more rapid NTM growth.

For sputum samples, there were 148 false-negative results; these samples were found to be negative using LiPA for MTBC detection but culture positive. Of these, 104 (70.2\%) specimens were smear positive representing 4\% (104 of 2606) of the total smear-positive sputum samples analysed, 28 (18.9\%) were smear negative representing $5.4 \%(28 / 523)$ of the total smear-negative sputum samples analysed and $16(10.8 \%)$ were unknown. There were 165 cases which appeared to be false-positive MTBC results using LiPA; these specimens were positive by PCR but failed to grow MTBC using standard culture techniques. Of the 165 false-positive results, $122(73.9 \%)$ specimens were smear positive representing $4.7 \%(122 / 2606)$ of the total smear-positive sputum samples analysed, 29 (17.6\%) were smear negative representing $5.5 \%(29 / 523)$ of the total smear-negative sputum samples analysed and 14 (8.5\%) were unknown.

There were 10 false-negative rifampicin sensitive results; 10 cases were reported as sensitive to rifampicin by PCR but the standard phenotypic sensitivity showed resistance. However, there were 14 false-positive results using LiPA. These 14 cases were reported as resistant to rifampicin using PCR but were subsequently considered to be sensitive to rifampicin using the standard phenotypic sensitivity. Of the 14 false-positive cases, $12(85.7 \%)$ were from respiratory specimens (table 2$)$.

Comparative analysis between 1999-2002 and 2003-2009 data The sensitivity, specificity, PPV and NPV were compared with our previously published data ${ }^{5}$ using the $\chi^{2}$ test. Data from the

Table 3 Days saved to reach earlier diagnosis by implementing the Innolipa LiPA Rif assay

\begin{tabular}{llll}
\hline & $\begin{array}{l}\text { All } \\
\text { samples }\end{array}$ & $\begin{array}{l}\text { Smear } \\
\text { positive only }\end{array}$ & $\begin{array}{l}\text { Smear } \\
\text { negative only }\end{array}$ \\
\hline $\begin{array}{l}\text { Days saved before culture results } \\
\text { (mean } \pm \text { standard deviation) }\end{array}$ & $35.85 \pm 24.89$ & $25.30 \pm 19.36$ & $47.57 \pm 26.32$ \\
$\begin{array}{l}\text { Days saved before rifampicin } \\
\text { sensitivity results (mean } \pm \text { standard } \\
\text { deviation) }\end{array}$ & $31.99 \pm 17.71$ & $32.15 \pm 16.90$ & $31.85 \pm 20.64$ \\
\hline
\end{tabular}

Tuberculosis assay in comparison to the standard culture and sensitivity techniques (2006-2009). 
current study (2003-2009) were in line with previously published data (1999-2002) apart from a significant increase in the sensitivity of the test for smear-negative samples in general and their subgroups, namely respiratory and non-respiratory groups (table 4). The improvement in sensitivity highlights the importance of operator experience in performing the LiPA assays.

\section{DISCUSSION}

We have had the ability to rapidly and accurately diagnose TB and rifampicin resistance in sputum microscopy smear-positive specimens since the 1990s. ${ }^{5}$ 14-17

In 2008, 8655 cases of TB were reported in the UK, representing an increase of $2.2 \%$ in the rate of disease compared with 2007. Mycobacterial TB isolates with drug sensitivity test results showed that $1.1 \%$ were MDRTB. ${ }^{18}$ Patients with MDRTB are difficult to manage, treatment is costly and must be administered for a long time, and drugs can be toxic. Drug toxicity and the length of treatment often contribute to non-compliance, which can increase the likelihood of developing extensively drug-resistant TB (XDRTB) (ie, MDRTB isolates that are also resistant to a quinolone and one or more of the injectable drugs-amikacin, capreomycin and kanamycin).

Routinely, specimens received at the NMRL are first cultured and then identified as MTBC using GenoType-Series molecular assay or DNA sequencing. The advantage of 'Fastrack' is that TB and MDRTB can be diagnosed within a day (permitting appropriate clinical, infection control and public health action, and improving patient outcomes ${ }^{2-4}$ ) and the specimens are subjected to rapid automated culture. Specimens known to have MDRTB produce cultures that can then be analysed for all firstline and reserve drugs simultaneously.

The overall sensitivity, specificity, PPV and NPV for MTBC detection in all specimens using LiPA were $87.3 \%, 91.1 \%, 87.7 \%$ and $90.8 \%$, respectively. These values are similar to those reported in our previous study at the beginning of the service, when overall sensitivity, specificity, PPV and NPV were $85.2 \%$, $88.2 \%, 86.9 \%$ and $86.7 \%$, respectively. ${ }^{5}$ As with other trials, ${ }^{5} 19$ sensitivity of PCR was higher in smear-positive respiratory samples $(93.8 \%)$ compared with smear-negative respiratory samples $(70.4 \%)$ and all non-respiratory samples $(71.4 \%)$. The lower sensitivity of PCR in these samples may be explained by lower mycobacterial loads, suboptimal sample volumes, and irregular clumping of organisms in paucibacillary specimens leading to sampling errors.

This study is a large operational study of using LPAs in a nontrial context over a decade. It demonstrates the reality of an operational service and that non-automated LPAs using well trained technical staff working to strict operating procedures achieves comparable sensitivity. At the NMRL, bacteriological staff performing this procedure usually had no prior experience of molecular diagnostic assays but were appropriately trained over a 1-week-period. A PhD grade molecular scientist oversaw the service.

The principal weakness of the study is its retrospective nature but the service was designed to analyse performance with the chosen parameters in mind. It measures the real performance of a national service under non-trial conditions based on NHS referrals by clinicians. The relatively lower specificity for TB detection in smear-positive specimens that had not grown in culture may be because specimens were taken while patients were on TB therapy. There was some circumstantial evidence that therapy had been started before specimens were taken. The request forms for 'Fastrack' asked if any treatment had been administered and in over two-thirds of forms for this patient

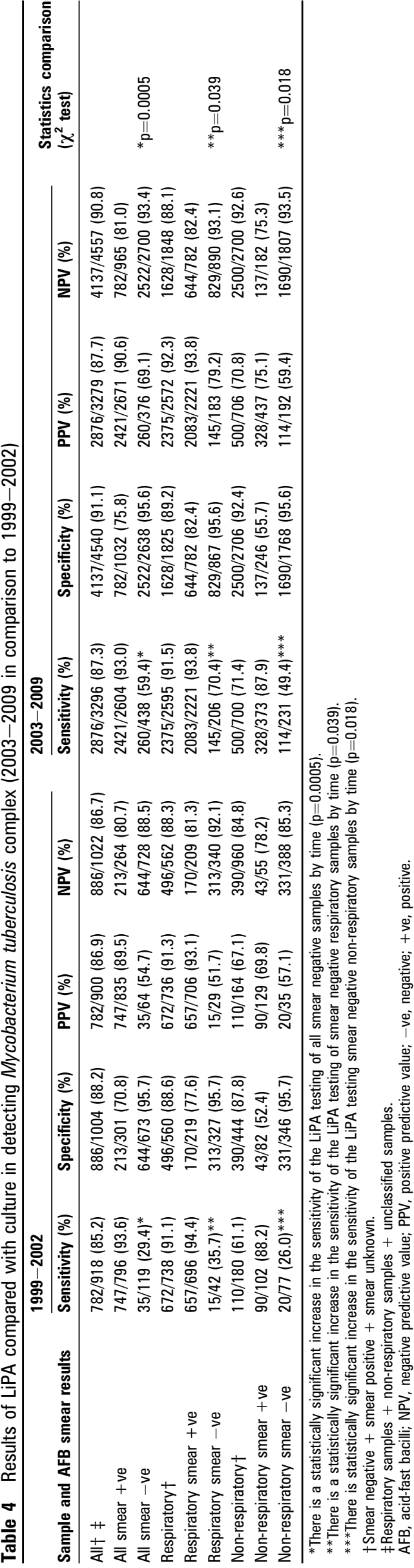


group the free text section indicated that the patient was on treatment. It is well documented that PCR-based techniques would detect DNA for dead bacteria but these bacteria would not be cultivable. This study was not designed to measure the actual impact on clinical management but supports other studies that suggest earlier diagnosis of MDRTB is advantageous. ${ }^{2}{ }^{20-22}$

The recent study by Boehme et al demonstrated a high sensitivity even for smear-negative sputum samples $(72.5 \%$ for single automated XpertMTB/RIF compared with culture) but we were able to show a comparable level of sensitivity for LPA, indicating that this remains a highly sensitive and specific tool in appropriate laboratories. The XpertMTB/RIF assay is simple to perform requiring limited training to achieve quality results but at a higher cost. LPA requires less proprietary equipment but a more appropriate PCR-suite infrastructure, longer training and greater operator expertise than XpertMTB/RIF. Having effective competitor assays will support cost reduction for XpertMTB/RIF, LPA and other assays under development.

As most parts of the world have a low prevalence of MDRTB this will lead to a low PPV. Globally, confirmation of positive XpertMTB/RIF (and/or LPA) results using another molecular method or with microbiological DST is needed to be in line with WHO policy recommendations. For maximum benefit, there should be rapid identification of TB cultures with phenotypic analysis of first-line and second-line DST once a molecular test has identified drug resistance mutations associated with rifampicin (and possibly isoniazid) resistance. This will significantly

\section{What is already known on this topic}

A recent study described the performance of a new automated diagnostic system (Cepheid, California, USA GeneXPert, XpertMTB/RIF) for tuberculosis (TB) and multidrug resistant TB (MDRTB). In that study the two features that stood out were the high sensitivity of the assay in patients who were sputum smear negative and the ease of use with minimal technical training required.

\section{What this study adds}

The authors describe the performance of a national UK diagnostic service for TB and rifampicin resistance in a non-trial context using another $\mathrm{WHO}$-endorsed non-automated approach based on line probe assays over the last decade. The authors feel this study complements other studies on the XpertMTB/RIF system, demonstrating a comparable sensitivity and clearly indicating that there is an alternative (and potentially cheaper) strategy for sensitive TB diagnosis. The study also demonstrates the realworld performance of such a diagnostic service which will assist policy makers and TB programme managers in determining the optimum configuration and roll-out of services. For example, the line probe assay requires much less (and mostly generic) laboratory equipment but is technically more demanding. For centres that have the technical skills, there is an effective alternative to the XpertMTB/RIF system and the resulting competition will support competitive pricing in low and middle income countries (and probably industrialised countries where TB services are part of the public sector).

\section{Limitations of the study}

Ideally additional clinical information would have been useful. However, it was previously shown that smear-positive specimens from patients who remained persistently positive on treatment (arousing suspicion that the patient has drug-resistant Mycobacterium tuberculosis complex or was not adherent to therapy) were frequently culture positive on therapy as the bacilli were viable because of resistance or due to patients' nonadherence to treatment. ${ }^{23}$ Therefore, not excluding patients currently on treatment might have reduced the specificity but not as much as might be expected.

reduce the time between sputum collection and full susceptibility testing for MDRTB cases. Addressing timeliness in technological improvement should go in tandem with minimising organisational delay. Clinicians need to make prompt therapeutic changes following rapid DST.

Assays with higher sensitivities are needed to address paucibacillary samples from patients who are HIV positive (despite the significant improvement demonstrated for the XpertMTB/ RIF assay in this patient group), children especially the very young who produce little or no sputum, and extrapulmonary material, particularly dilutional fluids such as CSF, ascites and pleural fluid.

\section{CONCLUSION}

A national molecular diagnostic service for TB and drug-resistant TB (using a non-automated LPA) provides a rapid and reliable national service for diagnosing MTBC and rifampicin resistance.

Acknowledgements We thank Dr Richard Hooper, Barts and the London School of Medicine, for valuable statistical advice. We would like to thank all the staff at the HPA NMRL for their assistance.

Funding The study was funded internally by the UK Health Protection Agency and Barts and the London School of Medicine, Queen Mary University.

Competing interests We declare that we have no conflict of interest. Specifically all the LiPA molecular and culture assays described were purchased at the normal price. All authors have completed the Unified Competing Interest form at http://www.icmje. org/coi disclosure.pdf (available on request from the corresponding author) and declare no support from any organisation for the submitted work; no financial relationships with any organisations that might have an interest in the submitted work in the previous 3 years; and no other relationships or activities that could appear to have influenced the submitted work.

Patient consent Uses routine data submitted on request form for TB diagnosis sent to our centre. Samples part of standard of care.

Ethics approval Uses routine data submitted on request form for TB diagnosis sent to our centre.

Contributors FAD, NS, SM and TB contributed to study design. NS, SM, TB, FD, AKA and FAD participated in data collection and NS, SM, TB and FAD in data management NS, SM, TB, FD, AKA and FAD contributed to data analysis. NS, SM and FAD wrote the manuscript; all other authors critically reviewed it and approved the final version.

Provenance and peer review Not commissioned; externally peer reviewed.

\section{REFERENCES}

1. Drobniewski FA, Balabanova YM. The diagnosis and management of multipledrug-resistant-tuberculosis at the beginning of the new millennium. Int $\mathrm{J}$ Infect Dis 2002;6(Suppl 1):S21-31.

2. Turett GS, Telzak EE, Torian LV, et al. Improved outcomes for patients with multidrug-resistant tuberculosis. Clin Infect Dis 1995;21:1238-44.

3. Drobniewski F, Eltringham I, Graham C, et al. A national study of clinical and laboratory factors affecting the survival of patients with multiple drug resistant tuberculosis in the UK. Thorax 2002;57:810-16. 
4. Choi JH, Lee KW, Kang HR, et al. Clinical efficacy of direct DNA sequencing analysis on sputum specimens for early detection of drug-resistant Mycobacterium tuberculosis in a clinical setting. Chest 2010;137:393-400.

5. Sam IC, Drobniewski F, More P, et al. Mycobacterium tuberculosis and rifampin resistance, United Kingdom. Emerg Infect Dis 2006;12:752-9.

6. WHO. Policy statement: Molecular line probe assays for rapid screening of patients at risk of multidrug-resistant tuberculosis (MDR-TB). Geneva: WHO, 2008.

7. Boehme CC, Nabeta P, Hillemann D, et al. Rapid molecular detection of tuberculosis and rifampin resistance. N Engl J Med 2010;363:1005-15.

8. Drobniewski FA. Diagnosing multidrug resistant tuberculosis in Britain. Clinical suspicion should drive rapid diagnosis. BMJ 1998;317:1263-4.

9. Barnard M, Albert H, Coetzee G, et al. Rapid molecular screening for multidrugresistant tuberculosis in a high-volume public health laboratory in South Africa. Am J Respir Crit Care Med 2008;177:787-92.

10. Kirehn TE, Cynamon MH, Inderlied CB, et al. National Committee for Clinical Laboratory Standards. Antimicrobial susceptibility testing for Mycobacterium tuberculosis; tentative standard NCCLS document M24-T. 1994:17:7-11.

11. Kruuner A, Yates MD, Drobniewski FA. Evaluation of MGIT 960-based antimicrobial testing and determination of critical concentrations of first- and second-line antimicrobial drugs with drug-resistant clinical strains of Mycobacterium tuberculosis. J Clin Microbiol 2006;44:811-18.

12. Rusch-Gerdes S, Pfyffer GE, Casal M, et al. Multicenter laboratory validation of the BACTEC MGIT 960 technique for testing susceptibilities of Mycobacterium tuberculosis to classical second-line drugs and newer antimicrobials. J Clin Microbiol 2006;44:688-92.

13. Vollset SE. Confidence intervals for a binomial proportion. Stat Med 1993;12:809-24.
14. Telenti A, Imboden P. Marchesi F, et al. Detection of rifampicin-resistance mutations in Mycobacterium tuberculosis. Lancet 1993;341:647-50.

15. De Beenhouwer $\mathbf{H}$, Lhiang Z, Jannes $\mathrm{G}$, et al. Rapid detection of rifampicin resistance in sputum and biopsy specimens from tuberculosis patients by PCR and line probe assay. Tuber Lung Dis 1995;76:425-30.

16. Skenders G, Fry AM, Prokopovica I, et al. Multidrug-resistant tuberculosis detection Latvia. Emerg Infect Dis 2005;11:1461-3.

17. Drobniewski FA, Caws M, Gibson A, et al. Modern laboratory diagnosis of tuberculosis. Lancet Infect Dis 2003:3:141-7.

18. HPA. Tuberculosis in the UK: annual report on tuberculosis surveillance in the UK Health Protection Agency Centre for Infections. London: HPA, 2009.

19. Sarmiento OL, Weigle KA, Alexander $\mathrm{J}$, et al. Assessment by meta-analysis of PCR for diagnosis of smear-negative pulmonary tuberculosis. J Clin Microbiol 2003;41:3233-40.

20. Drobniewski F, Balabanova Y, Coker R. Clinical features, diagnosis, and management of multiple drug-resistant tuberculosis since 2002. Curr Opin Pulm Med 2004:10:211-17

21. Leimane V, Dravniece G, Riekstina V, et al. Treatment outcome of multidrug/extensively drug-resistant tuberculosis in Latvia, 2000-2004. Eur Respir J 2010;36:584-93.

22. Nathanson E, Lambregts-van Weezenbeek C, Rich ML, et al. Multidrug-resistant tuberculosis management in resource-limited settings. Emerg Infect Dis 2006;12:1389-97.

23. Drobniewski FA, Watterson SA, Wilson SM, et al. A clinical, microbiological and economic analysis of a national service for the rapid molecular diagnosis of tuberculosis and rifampicin resistance in Mycobacterium tuberculosis. $\mathrm{J} \mathrm{Med}$ Microbiol 2000;49:271-8.

Visit Thorax online and listen to the latest podcast, post comments and download any you might have missed. Keep informed and up to date by visiting thorax.bmj.com. 